\title{
Repeated Dry Sauna Therapy Improves Quality of Life in Obese Korean People
}

\author{
Hoon Sung Choi', Hyuki Kwon', Keun-Hyok Cho' ${ }^{1}$ Le Cong Thuc', So Young Park', Nam-Hun Kim² , Wae-Jung Kim², \\ Yun-Ki Kim², Woo-Seok Jeon'², Ju-Ah Lee', Hyoung-Chun Kim ${ }^{3}$, Eun-Hee Cho ${ }^{1, *}$ \\ 'Department of Internal Medicine, Kangwon National University School of Medicine, Chuncheon, Korea \\ ${ }^{2}$ College of Forest and Environmental Sciences, Kangwon National University, Chuncheon, Korea \\ ${ }^{3}$ Neuropsychopharmacology and Toxicology Program, College of Pharmacy, Kangwon National University, Chuncheon, Korea
}

Background: Dry sauna treatments improve the quality of life for chronic pain, congestive heart failure, and type 2 diabetes patients. This study aimed to determine whether dry sauna therapy improved the quality of life of obese people.

Methods: A total of 38 consecutive participants aged over 20 years with a body mass index of $\geq 25 \mathrm{~kg} / \mathrm{m}^{2}$ were recruited for the study. The participants were treated with a $90^{\circ} \mathrm{C}$ dry sauna for 15 minutes, twice daily for 4 consecutive days. To assess the quality of life, all participants completed the 5 level EQ-5D questionnaires and the EQ-Visual Analog Scale. Study parameters were measured on the same day prior to commencing the sauna sessions in a fasted state and 2 days after the last sauna session.

Results: The average age was $62.3 \pm 9.5$ years; $84.2 \%$ of the participants were female. The mean body mass index was $28.5 \pm 2.4 \mathrm{~kg} / \mathrm{m}^{2}$. Dry sauna significantly improved the mean 5 level EQ-5D index scores from $0.83 \pm 0.12$ to $0.89 \pm 0.11$ and increased the mean EQ-Visual Analog Scale from 79.0 \pm 15.2 to 91.1 \pm 9.7 . However, there were no significant changes in body mass index, blood pressure, heart rate, or body composition before and after the 8-session sauna therapy.

Conclusion: Dry sauna improved the health-related quality of life of obese patients without adverse events. Further clinical studies in larger study populations are needed to verify these findings and provide concrete evidence for obesity treatment.

Keywords: Sauna; Quality of Life; Obesity; Infrared Rays; Korea

Received: September 12, 2018, Revised: November 16, 2018, Accepted: November 22, 2018

*Corresponding Author: Eun-Hee Cho https://orcid.org/0000-0002-1349-8894

Tel: +82-33-258-9167, Fax: +82-33-258-2455, E-mail: ehcho@kangwon.ac.kr 


\section{INTRODUCTION}

In many countries including Korea and Japan, dry sauna has been popular for hundreds of years as an alternative therapy for people who want to improve their health conditions without relying on pharmaceuticals. However, there has been little clinical evidence of its effectiveness until recently.

Some studies have shown that repeated sauna therapy improved not only the cardiac, endothelial, and exercise function but also the quality of life (QOL) of patients with chronic heart failure in Japan. ${ }^{1-3)}$ Other studies have also reported that repeated sauna therapy improved peripheral arterial disease, ${ }^{4)}$ exercise tolerance, and the QOL in patients with severe chronic obstructive pulmonary disease. ${ }^{5)}$

Over the past decades, the prevalence of obesity has rapidly increased worldwide. This raises concern about the burden of diseases (such as diabetes, cancers, and cardiovascular diseases) and their effect on QOL. Previous studies have shown that obese individuals have poorer QOL than non-obese controls. ${ }^{6,7)}$ A study has also reported that obese individuals had a significantly lower health-related quality of life (HRQoL) than individuals that were within the normal weight range in the United States general population. ${ }^{8)}$ Another study has also suggested that obese women with fractures had a significantly lower QOL than non-obese participants with fractures. ${ }^{9)}$ According to the Korea National Health and Nutrition Examination Survey, there was a negative association between obesity and HRQoL due to obesity-related diseases in both men and women. ${ }^{10)}$ Therefore, the relationship between obesity and HRQoL clearly deserves a close attention. To the best of our knowledge, there is no published research that has examined the effects of dry sauna therapy on the QOL of obese people. The objective of this study was to investigate whether dry sauna may improve the HRQoL in obese individuals who do not present with the obesity-related diseases observed in general medical practice.

\section{METHODS}

\section{Selection of Participants}

A total of 38 consecutive participants who met the inclusion criteria were enrolled in the study between December 2016 and February 2017 in Jincheon, Korea. The inclusion criteria were patients aged over 20 years, with a body mass index (BMI) of $\geq 25.0 \mathrm{~kg} / \mathrm{m}^{2}$. Diabetic patients were included in our study; however, we excluded patients who had severe acute hyperglycemic complications, such as diabetic ketoacidosis or hyperglycemic hyperosmolar state. The exclusion criteria were participants with severe liver disease, congestive heart failure, or chronic kidney disease; participants who had a history of myocardial infarction or cerebral infarction; women who were pregnant or lactating; and participants with a major comorbidity such as cancer. A selfadministered questionnaire, interviews, and anthropometric measurements including previous medical history were used to obtain data from the participants. Baseline data from the questionnaires, anthropometric measurements, fasting blood samples, and body com- position analyses were obtained on the same morning prior to the first dry sauna session (session 1). The above data were also obtained 2 days (due to snowfall) after the last dry sauna therapy (session 8).

\section{Dry Sauna Therapy}

Dry sauna therapy (Backgok, Jincheon, Korea), a form of thermal therapy, employs a far-infrared dry sauna at $90^{\circ} \mathrm{C}$ using a wooden stove and is performed without hydration. The dry sauna therapy was performed twice daily for 4 consecutive days, with a total of eight sessions. Each sauna session consisted of a 15-minute exposure to dry sauna at $90^{\circ} \mathrm{C}$ and a 30 -minute rest at room temperature $\left(24^{\circ} \mathrm{C}\right)$. The weight and blood pressure were checked for all participants before and after every session. Oral rehydration (with water) was encouraged to compensate for any weight loss due to perspiration.

\section{Primary Outcome Measurement}

The 5 level EQ-5D (EQ-5D-5L) index score and EQ-Visual Analog Scale (VAS) were used to measure the HRQoL of the participants. The patients were asked to answer questionnaires (EQ-5D-5L index score) consisting of five dimensions (mobility, self-care, usual activities, pain/ discomfort, and anxiety/depression) by skilled research nurses. Each dimension had five levels (no problems, slight problems, moderate problems, severe problems, and extreme problems). The EQ-VAS recorded the respondent's self-rated health on a vertical, VAS (the endpoints were labeled as 'best imaginable health state, 100' and 'worst imaginable health state, 0 '). The EQ-5D-5L and EQ-VAS questionnaires were administered two days prior to the first sauna session and one day after the last sauna session.

\section{Secondary Outcome Measurements}

Secondary outcome variables, including height, body weight, body composition, blood pressures, complete blood count, and admission battery were measured before (session 0 ) and after sauna therapy (ses-

Table 1. Clinical characteristics of study participants

\begin{tabular}{lc}
\multicolumn{1}{c}{ Characteristic } & Value \\
\hline No. of participants & 38 \\
Age $(\mathrm{y})$ & $62.3 \pm 9.5$ \\
Sex & $6(15.8)$ \\
Male & $32(84.2)$ \\
Female & $15(39.5)$ \\
Hypertension & $5(13.2)$ \\
Diabetes & $3(7.9)$ \\
Dyslipidemia & \\
Smoking history & $31(81.6)$ \\
Non-smoker & $6(15.8)$ \\
Ex-smoker & $1(2.6)$ \\
Currently smoking & $28.5 \pm 2.4$ \\
Body mass index $\left(\mathrm{kg} / \mathrm{m}^{2}\right)$ & $90.1 \pm 7.2$ \\
Waist circumference $(\mathrm{cm})$ & $136.5 \pm 17.2$ \\
Systolic blood pressure $(\mathrm{mm} \mathrm{Hg})$ & $80.8 \pm 10.3$ \\
Diastolic blood pressure $(\mathrm{mm} \mathrm{Hg})$ &
\end{tabular}

Values are presented as number, mean \pm standard deviation, or number (\%). 
sion 8). Anthropometric measurements, including body composition using InBody 520 (Biospace, Seoul, Korea) and fasting blood plasma samples, and clinical information relevant to obesity were obtained from each participant before (session 0 ) and after therapy (session 8). Fasting venous blood samples were obtained from each participant two days before the first session and one day after the last session. These blood samples were used to measure biochemical parameters such as glucose, hemoglobin Alc (HbAlc), lipid profile, protein, albumin, blood urea nitrogen, creatinine, aspartate transaminase, alanine transaminase, and uric acid levels.

\section{Statistical Analysis}

The results are expressed as the mean \pm standard deviation. The differences in parametric values before (session 0 ) and after (session 8) dry sauna therapy were analyzed using the paired Student t-test. Nonparametric pairwise comparisons were made using the Wilcoxon rank-sum test. Multivariate analyses were performed by linear regression analysis with adjustment for age, sex, and the presence of diabetes. All P-values are two-tailed, and a P-value of $<0.05$ was considered as statistically significant. The statistical analyses were performed using the IBM SPSS ver. 22.0 software program (IBM Corp., Armonk, USA).

\section{Ethics Statement}

This study was approved by the institutional review board of Kangwon National University Hospital (IRB approval no., 2016-11-009). A written informed consent was obtained from each participant.

\section{RESULTS}

\section{Characteristics of Individuals with Obesity}

Table 1 shows clinical characteristics of the 38 obese participants in this study. There was a higher percentage of females $(84.2 \%)$ than males $(15.8 \%)$ in this study. The average age was $62.3 \pm 9.5$ years (range, 35-72 years). Out of the 38 participants, 15 (39.5\%) had hypertension,
5 (13.2\%) had type 2 diabetes, and $31(81.6 \%)$ were nonsmokers. The mean BMI was $28.5 \pm 2.4 \mathrm{~kg} / \mathrm{m}^{2}$ and the mean waist circumference was $90.1 \pm 7.2 \mathrm{~cm}$.

\section{Comparisons between Clinical Parameters, Quality of Life, and Body Composition before and after Dry Sauna Therapy in Participants}

The effects of the eight-session dry sauna therapy on clinical parameters, QOL and body composition of the study participants are summarized in Table 2. There were no changes in blood pressure, heart rate, and BMI after the eight-session dry sauna therapy. Figure 1 shows that dry sauna significantly improved the mean EQ-5D-5L index scores from $0.83 \pm 0.12$ to $0.89 \pm 0.11$ and increased the mean EQ-VAS scores from $79.0 \pm 15.2$ to $91.1 \pm 9.7$. Among the components of EQ-5D-5L index, there were significant improvements in pain/discomfort and anxiety/depression ( $\mathrm{P}=0.002$ and $\mathrm{P}=0.047$, respectively). However, there were no significant changes in $\mathrm{BMI}$, blood pressure, heart rate, and
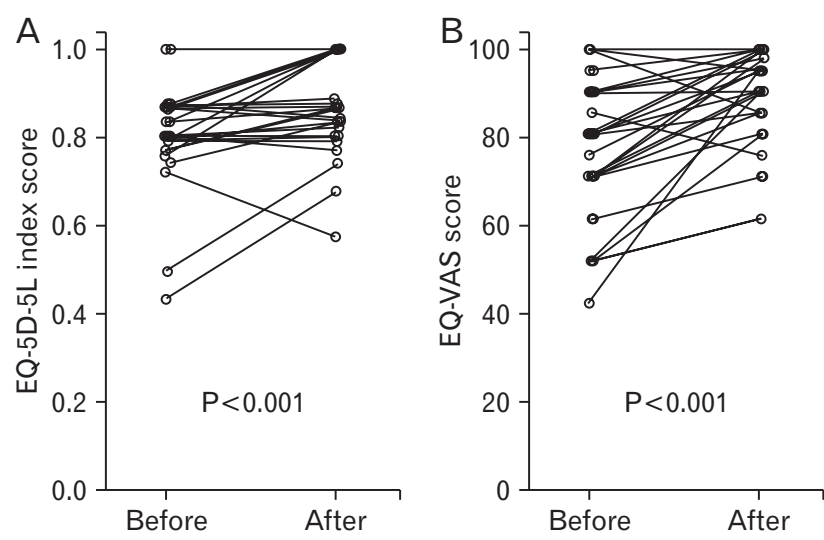

Figure 1. Changes in individual percentages of the EQ-5D-5L index score (\% flow mediated dilation) and EQ-VAS score before and after 8 sessions of dry sauna therapy. (A) Individual changes of EQ-5D-5L index score after dry sauna compared to baseline score. (B) Individual changes of EQ-VAS score after dry sauna. Paired t-test showed a significant improvement in both parameters. EQ-5D-5L, 5 level EQ5D; EQ-VAS, EQ-Visual Analog Scale.

Table 2. Comparison of clinical parameters, quality of life, and body composition data of obese participants before and after dry sauna

\begin{tabular}{|c|c|c|c|c|}
\hline Variable & Before dry sauna & After dry sauna & P-value* & P-value \\
\hline No. of patients & 38 & 38 & & \\
\hline Body mass index $\left(\mathrm{kg} / \mathrm{m}^{2}\right)$ & $28.5 \pm 2.4$ & $28.4 \pm 2.5$ & 0.241 & 0.883 \\
\hline Waist circumference (cm) & $90.1 \pm 7.2$ & $89.7 \pm 6.8$ & 0.472 & 0.756 \\
\hline Systolic blood pressure (mm Hg) & $136.5 \pm 17.2$ & $134.3 \pm 16.1$ & 0.307 & 0.544 \\
\hline Diastolic blood pressure $(\mathrm{mm} \mathrm{Hg})$ & $80.8 \pm 10.3$ & $78.8 \pm 9.9$ & 0.109 & 0.347 \\
\hline Heart rate (beats/min) & $73.5 \pm 8.9$ & $72.3 \pm 10.3$ & 0.182 & 0.570 \\
\hline Total body water (\%) & $33.5 \pm 5.7$ & $34.4 \pm 7.4$ & 0.437 & 0.431 \\
\hline Skeletal muscle mass $(\mathrm{kg})$ & $25.5 \pm 5.8$ & $25.9 \pm 5.3$ & 0.724 & 0.719 \\
\hline Body fat mass (kg) & $24.2 \pm 8.8$ & $22.9 \pm 8.6$ & 0.424 & 0.454 \\
\hline Percent body fat (\%) & $34.2 \pm 11.4$ & $32.5 \pm 11.4$ & 0.477 & 0.439 \\
\hline 5 Level EQ-5D index score & $0.83 \pm 0.12$ & $0.89 \pm 0.11$ & $<0.001$ & 0.003 \\
\hline EQ-Visual Analog Scale & $79.0 \pm 15.2$ & $91.1 \pm 9.7$ & $<0.001$ & $<0.001$ \\
\hline
\end{tabular}

Values are presented as number or mean \pm standard deviation.

${ }^{*}$ Calculated by the paired Student t-test for parametric analysis and the Wilcoxon rank-sum test for non-parametric analysis. ${ }^{\dagger}$ Calculated by linear regression model, with adjustment for age, sex, and the presence of diabetes. 
body composition before and after the eight-session dry sauna therapy. Multivariate analyses showed corresponding results with adjustment for age, sex, and the presence of diabetes.

\section{Biochemical Changes in Participants, before and after Dry Sauna Therapy}

After eight sessions of the dry sauna therapy, there was no change in the liver and renal functions of participants. Also, there was no significant change in fasting glucose, total serum cholesterol, triglycerides, high-density lipoprotein cholesterol, and uric acid concentrations. However, there was a significant increase in HbAlc and a significant decrease in albumin levels after the dry sauna. Apart from these parameters, no significant differences were observed in other biochemical parameters before and after the dry sauna therapy (Table 3). Multivariate analyses showed corresponding results with adjustment for age, sex, and the presence of diabetes.

\section{Adverse Events}

There were no adverse events before, during, and after the dry sauna therapy in all participants. Specifically, there were no reported cases of syncope, heat exhaustion, or heat stroke.

\section{DISCUSSION}

Sauna therapy has been traditionally utilized for years in many cultures. Previous studies have shown that sauna therapy is safe for therapeutic purposes and offers health benefits primarily with regard to diabetes $^{11)}$ and cardiovascular diseases. ${ }^{12-16)}$ However, most of these studies had small sample sizes with short study durations; hence, their results needed confirmation and could not be generalized to other clinical areas. ${ }^{17)}$ Even though our study also had a small sample size, we found that repeated dry sauna therapy significantly improved the HRQoL of obese participants without any adverse effects. One of the strengths of this study was that it did not rely on inpatients as participants. In this study, 38 otherwise healthy obese participants were recruited and participated till the end of the study. The $100 \%$ completion rate for the dry sauna therapy is significant in predicting the successful acceptance and adherence to lifestyle changes, which suggests that dry sauna is a valuable adjunctive lifestyle option.

The sauna cabin was heated to about $300^{\circ} \mathrm{C}$ to $400^{\circ} \mathrm{C}$. The emission was in the far-infrared radiation (FIR) range, which had biological effects on the human body. ${ }^{18)}$ Despite these beneficial applications, the exact molecular mechanisms in response to the sauna and the biological effects of FIR as well as the proper timing, frequency, and temperature of sauna for maximal efficacy are still poorly understood. A previous study has reported of a significant improvement in the physical and mental QOL in Japanese patients with congestive heart failure. ${ }^{2)}$ The study suggests that mild warming could be a possible mechanism for dry saunas that sedated the sensory nerve endings and increased the level of $\beta$-endorphins. ${ }^{19-21)}$ In most Japanese studies, a $60^{\circ} \mathrm{C}$ dry sauna was applied for 15 minutes once daily., ${ }^{2,12,15,18,22,23)}$ However, Koreans generally prefer dry saunas at a higher temperature for prolonged periods; thus, we applied a $90^{\circ} \mathrm{C}$ dry sauna for 15 minutes twice daily. From our study, dry sauna therapy at $90^{\circ} \mathrm{C}$ could be safely performed in obese people without any adverse effects.

Our results showed a significant increase in $\mathrm{HbAlc}$ and a significant decrease in albumin levels after dry sauna, compared with before dry sauna. The mechanism underlying these significant changes in $\mathrm{HbAlc}$ and albumin after dry sauna is not clear. The increase of HbAlc seems to be related to dehydration or hemoconcentration after dry sauna. ${ }^{22-24)}$ The change in protein or albumin levels after thermal stress is controversial and may have been influenced by the time of measurement after dry sauna, degree of rehydration, and sex. ${ }^{25-27)}$ Further studies are needed to clarify the precise mechanisms underlying the observed biochemical changes after dry sauna therapy.

Our study had several limitations. First, the sample size was small,

Table 3. A comparison of the biochemical of obese participants values before and after dry sauna

\begin{tabular}{|c|c|c|c|c|}
\hline Variable & Before dry sauna & After dry sauna & P-value* & P-value ${ }^{\dagger}$ \\
\hline No. of participants & 38 & 38 & & \\
\hline Hemoglobin A1c & $5.9 \pm 0.9$ & $6.0 \pm 0.9$ & 0.027 & 0.835 \\
\hline Protein & $7.1 \pm 0.4$ & $7.2 \pm 0.3$ & 0.106 & 0.190 \\
\hline Albumin & $4.6 \pm 0.2$ & $4.4 \pm 0.2$ & $<0.001$ & 0.001 \\
\hline Total bilirubin & $0.5 \pm 0.2$ & $0.5 \pm 0.2$ & 0.961 & 0.978 \\
\hline Aspartate transaminase & $25.3 \pm 6.2$ & $26.3 \pm 8.4$ & 0.305 & 0.516 \\
\hline Alanine transaminase & $25.5 \pm 12.3$ & $26.8 \pm 13.2$ & 0.273 & 0.610 \\
\hline Blood urea nitrogen & $15.0 \pm 4.6$ & $16.0 \pm 4.2$ & 0.081 & 0.299 \\
\hline Creatinine & $0.8 \pm 0.2$ & $0.8 \pm 0.2$ & 0.753 & 0.893 \\
\hline Total cholesterol & $190.2 \pm 36.2$ & $189.0 \pm 34.6$ & 0.626 & 0.860 \\
\hline Triglyceride & $141.0 \pm 67.7$ & $150.6 \pm 120.1$ & 0.435 & 0.652 \\
\hline High-density lipoprotein cholesterol & $48.7 \pm 10.7$ & $48.4 \pm 11.7$ & 0.683 & 0.890 \\
\hline Low-density lipoprotein cholesterol & $121.5 \pm 33.0$ & $120.0 \pm 30.1$ & 0.616 & 0.842 \\
\hline Uric acid & $4.7 \pm 1.2$ & $4.7 \pm 1.2$ & 0.287 & 0.708 \\
\hline
\end{tabular}

Values are presented as number or mean \pm standard deviation.

${ }^{*}$ Calculated by the paired Student $\mathrm{t}$-test for parametric analysis and the Wilcoxon rank-sum test for non-parametric analysis. ${ }^{\dagger}$ Calculated by linear regression model, with adjustment for age, sex, and the presence of diabetes. 
and the study protocol employed a before and after therapy model without a control group. Our results cannot be generalized because the study had a higher percentage of women than men and the participants were all older Koreans. Second, the study period was relatively short. The study lasted for only eight sessions (15 minutes per session) for 4 consecutive days. Third, the EQ-5D-5L index score and the EQVAS used to assess the HRQoL were based on individual subjective feelings hence it lacked sufficient validity and reliability. Therefore, the effectiveness of dry sauna therapy may be better examined using blinded placebo-controlled studies with larger numbers of participants and longer study periods in an outpatient setting.

In conclusion, our results suggest that dry sauna therapy may be a useful and safe option for improving the HRQoL of obese patients. Nevertheless, further clinical studies in larger study populations are needed to verify these findings to give concrete evidence for the treatment of obesity.

\section{CONFLICT OF INTEREST}

No potential conflict of interest relevant to this article was reported.

\section{ACKNOWLEDGMENTS}

This study was supported by the Korea Forest Service grant (S111616 L040110).

\section{ORCID}

Hoon Sung Choi: https://orcid.org/0000-0002-7202-7777

Hyuki Kwon: https://orcid.org/0000-0002-3801-6273

Keun-Hyok Cho: https://orcid.org/0000-0003-4419-5578

Le Cong Thuc: https://orcid.org/0000-0002-5000-0020

So Young Park: https://orcid.org/0000-0003-1518-2516

Nam-Hun Kim: https://orcid.org/0000-0002-4416-0554

Yun-Ki Kim: https://orcid.org/0000-0002-0499-0246

Woo-Seok Jeon: https://orcid.org/0000-0002-3746-0815

Ju-Ah Lee: https://orcid.org/0000-0002-7823-8196

Hyoung-Chun Kim: https://orcid.org/0000-0003-0420-795X

Eun-Hee Cho: https://orcid.org/0000-0002-1349-8894

\section{REFERENCES}

1. Ohori T, Nozawa T, Ihori H, Shida T, Sobajima M, Matsuki A, et al. Effect of repeated sauna treatment on exercise tolerance and endothelial function in patients with chronic heart failure. Am J Cardiol 2012;109: 100-4.

2. Sobajima M, Nozawa T, Fukui Y, Ihori H, Ohori T, Fujii N, et al. Waon therapy improves quality of life as well as cardiac function and exercise capacity in patients with chronic heart failure. Int Heart J 2015;56: 203-8.

3. Miyata M, Kihara T, Kubozono T, Ikeda Y, Shinsato T, Izumi T, et al. Beneficial effects of Waon therapy on patients with chronic heart fail- ure: results of a prospective multicenter study. J Cardiol 2008;52:79-85. 4. Tei C, Shinsato T, Miyata M, Kihara T, Hamasaki S. Waon therapy improves peripheral arterial disease. J Am Coll Cardiol 2007;50:2169-71.

5. Umehara M, Yamaguchi A, Itakura S, Suenaga M, Sakaki Y, Nakashiki $\mathrm{K}$, et al. Repeated Waon therapy improves pulmonary hypertension during exercise in patients with severe chronic obstructive pulmonary disease. J Cardiol 2008;51:106-13.

6. Carpiniello B, Pinna F, Pillai G, Nonnoi V, Pisano E, Corrias S, et al. Psychiatric comorbidity and quality of life in obese patients: results from a case-control study. Int J Psychiatry Med 2009;39:63-78.

7. Sarac F, Paryldar S, Duman E, Saygyly F, Tuzun M, Yylmaz C. Quality of life for obese women and men in Turkey. Prev Chronic Dis 2007;4:A50.

8. Jia H, Lubetkin EI. The impact of obesity on health-related quality-oflife in the general adult US population. J Public Health (Oxf) 2005;27: 156-64.

9. Copes RM, Dal Osto LC, Langer FW, de Vieira AR, Codevilla AA, Sartori GR, et al. Low health related quality of life associated with fractures in obese postmenopausal women in Santa Maria, Brazil. Bone Rep 2017;6:70-3.

10. Park S. Pathways linking obesity to health-related quality of life. Qual Life Res 2017;26:2209-18.

11. Beever R. The effects of repeated thermal therapy on quality of life in patients with type II diabetes mellitus. J Altern Complement Med 2010;16:677-81.

12. Fujita S, Ikeda Y, Miyata M, Shinsato T, Kubozono T, Kuwahata S, et al. Effect of Waon therapy on oxidative stress in chronic heart failure. Circ J 2011;75:348-56.

13. Imamura M, Biro S, Kihara T, Yoshifuku S, Takasaki K, Otsuji Y, et al. Repeated thermal therapy improves impaired vascular endothelial function in patients with coronary risk factors. J Am Coll Cardiol 2001; 38:1083-8.

14. Kihara T, Biro S, Imamura M, Yoshifuku S, Takasaki K, Ikeda Y, et al. Repeated sauna treatment improves vascular endothelial and cardiac function in patients with chronic heart failure. J Am Coll Cardiol 2002; 39:754-9.

15. Kihara T, Miyata M, Fukudome T, Ikeda Y, Shinsato T, Kubozono T, et al. Waon therapy improves the prognosis of patients with chronic heart failure. J Cardiol 2009;53:214-8.

16. Sobajima M, Nozawa T, Ihori H, Shida T, Ohori T, Suzuki T, et al. Repeated sauna therapy improves myocardial perfusion in patients with chronically occluded coronary artery-related ischemia. Int J Cardiol 2013;167:237-43.

17. Beever R. Far-infrared saunas for treatment of cardiovascular risk factors: summary of published evidence. Can Fam Physician 2009;55: 691-6.

18. Vatansever F, Hamblin MR. Far infrared radiation (FIR): its biological effects and medical applications. Photonics Lasers Med 2012;4:255-66.

19. Glaser EM, Shephard RJ. Simultaneous experimental acclimatization to heat and cold in man. J Physiol 1963;169:592-602.

20. Kubota K, Kurabayashi H, Tamura K, Kawada E, Tamura J, Shirakura T. A transient rise in plasma beta-endorphin after a traditional $47 \mathrm{de}$ grees C hot-spring bath in Kusatsu-spa, Japan. Life Sci 1992;51:187780.

21. Vescovi PP, Gerra G, Pioli G, Pedrazzoni M, Maninetti L, Passeri M. Circulating opioid peptides during thermal stress. Horm Metab Res 
1990;22:44-6.

22. Lapolla A, Mosca A, Fedele D. The general use of glycated haemoglobin for the diagnosis of diabetes and other categories of glucose intolerance: still a long way to go. Nutr Metab Cardiovasc Dis 2011;21:46775.

23. Goth L. Analytical problems in determination of hemoglobin Alc and the different ways of its interpretation. Orv Hetil 2009;150:747-51.

24. Ernst E, Strziga P, Schmidlechner C, Magyarosy I. Sauna effects on hemorheology and other variables. Arch Phys Med Rehabil 1986;67: 526-9.
25. Rocker L, Kirsch K, Stoboy H, Schmidt HM, Wicke J. The influence of heat stress on plasma volume and intravascular proteins in sedentary females. Eur J Appl Physiol Occup Physiol 1977;36:187-92.

26. Ohira Y, Girandola RN, Simpson DR, Ikawa S. Responses of leukocytes and other hematologic parameters to thermal dehydration. J Appl Physiol Respir Environ Exerc Physiol 1981;50:38-40.

27. Rocker L, Kirsch KA, Stoboy H. Sex-dependent changes in plasma globulins in women and men exposed to heat stress. Isr J Med Sci 1978;14:212-7. 